\title{
Systematic review of the effect of probiotics on experimental gingivitis in humans
}

\author{
Eliane Porto BARBOZA ${ }^{(a)}$ \\ Patricia Carvalho ARRIAGA(a) \\ Diogo Pereira LUZ(a) \\ Caroline MONTEZ ${ }^{(a)}$ \\ Katia Costa VIANNA(a) iD \\ (a) Universidade Federal Fluminense - UFF, \\ School of Dentistry, Department of \\ Periodontology, Niterói, RJ, Brazil.
}

Declaration of Interests: The authors certify that they have no commercial or associative interest that represents a conflict of interest in connection with the manuscript.

\section{Corresponding Author:}

Eliane Porto Barboza

E-mail: elianeporto.uff@gmail.com

https://doi.org/10.1590/1807-3107bor-2020.vol34.0031

Submitted: May 23, 2019

Accepted for publication: September 1, 2019 Last revision: November 5, 2019

\begin{abstract}
Probiotic therapy is a viable alternative to chlorhexidine, a widely used antiseptic in dentistry that produces significant adverse effects. This systematic review aimed to analyze the effects of probiotics on experimental gingivitis in humans. Two independent reviewers conducted a comprehensive literature search until March 2019. Randomized clinical trials and controlled clinical trials were selected. Outcome data were extracted and critically analyzed. A total of five articles were included in the qualitative synthesis. No meta-analysis could be conducted due to the heterogeneity of the selected studies. The use of probiotics showed a slight improvement in clinical parameters. Changes in gingival crevicular fluid volume were lower in the presence of the probiotic than in the placebo group. All the studies showed that the immediate, positive effects of probiotics during the period of discontinued mechanical oral hygiene were due to the modulation of the host response, not the anti-plaque effect. Investigators should conduct randomized clinical trials to elucidate the mechanisms of probiotic action and develop improved delivery systems.
\end{abstract}

Keywords: Immunomodulation; Inflammation; Gingival Crevicular Fluid; Microbiota; Probiotics.

\section{Introduction}

The use of chemical agents in bacterial plaque control has been recommended for patients with greater susceptibility to gingivitis. ${ }^{1}$ However, prolonged use of antiseptics may be associated with side effects such as tooth staining, taste alteration and mucosal desquamation. ${ }^{2}$ Probiotics are live microorganisms which, when given in adequate amounts confer a health benefit on the host. ${ }^{3}$ They have shown promising potential as an alternative to mouthwashes that can cause undesired effects.

Dental caries and periodontal disease are the most common infectious diseases in humans, with approximately $90 \%$ prevalence. ${ }^{4}$ Recently, there has been an increasing interest in using probiotics to manage oral infections, and some controlled clinical studies have been conducted to elucidate the potential impact of probiotics on oral health. ${ }^{5,6,78}$ According to a study, probiotic intervention in childhood reduced salivary mutans streptococci and decreased the risk of dental caries, ${ }^{5}$ Krasse et al. ${ }^{6}$ showed a significant decrease in gingival bleeding with the administration of probiotic Lactobacillus 
reuteri. Oral administration of Lactobacillus salivarius WB21 reduced the levels of periodontopathic bacteria and improved periodontal status. ${ }^{7}$ The adjunctive use of probiotic tablets effectively inhibited periodontal pathogens and reduced inflammation in patients with gingivitis. ${ }^{8}$ However, the consumption of a probiotic milk beverage containing Lactobacillus casei strain Shirota showed no significant difference in experimental gingivitis between test and control groups. ${ }^{9}$

Although some studies with probiotics did not show improvements in the clinical parameters associated with gingivitis, they were able to demonstrate biomarker responses and modulate the activity of inflammatory cytokines, suggesting that host response could be regulated with the use of probiotics. ${ }^{6,9,10,11}$

Probiotics may be a suitable alternative to chlorhexidine, a widely used antiseptic in dentistry with potential side effects. Moreover, it has been predicted that the market for probiotic-containing supplements will grow from $\$ 35$ billion to $\$ 48$ billion by $2020 .^{12}$ This study aimed to systematically review randomized clinical trials on the effect of probiotics on experimental gingivitis.

\section{Methodology}

\section{Protocol registration}

This study was registered in PROSPERO (CRD42018116873). The Systematic Review followed PRISMA-P guidelines. ${ }^{13}$ The SR methodology followed the recommendations of the Cochrane Handbook for Systematic Reviews of Interventions. ${ }^{14}$ PRISMA checklist was used to ensure the quality and transparency of the study. ${ }^{15}$ The PICOS strategy was used to construct a focused question. ${ }^{16}$

\section{Focused question}

What are the effects of probiotics on clinical and biomolecular signs of inflammation in human experimental gingivitis?

\section{Clinical relevance}

Probiotics may be a useful therapeutic alternative to facilitate post-operative healing, as opposed to antimicrobial products.

\section{Search strategy}

A systematic search without date or language restrictions was performed using electronic databases such as PubMed/MEDLINE, Cochrane Library (CENTRAL), Web of Science and Trip until March 2019. A literature search of the following journals was conducted to complement the electronic search: Journal of Periodontology, Journal of Clinical Periodontology, Journal of Periodontal Research, The International Journal of Periodontics and Restorative Dentistry, and Clinical Oral Investigations. A search of the Grey Literature Report ${ }^{17}$ and OpenGrey databases ${ }^{18}$ revealed unpublished studies (grey literature). Finally, the reference/bibliography lists of all full-text articles (cross-referencing) and the ClinicalTrials.gov database were searched.

\section{Eligibility criteria based on PICOS strategy}

The present SR included studies that analyzed the effect of probiotics on the outcomes of experimental gingivitis (Table 1).

Population: Human adults presenting experimental gingivitis.

Intervention: use of probiotic therapy.

Comparison: use of placebo.

Outcomes: the primary outcome was gingivitis identified and graded by bleeding on probing (BOP), plaque index (PI), and gingival index (GI). The secondary outcome was the inflammatory response determined by gingival crevicular fluid (GCF) volume and biomarkers.

Study design: Randomized Clinical Trials (RCTs) and Clinical Controlled Trials (CCTs).

\section{Selection criteria}

This review sought RCTs and a CCT comparing the effect of probiotics on experimental gingivitis in humans. Animal studies, retrospective cohort studies, in vitro studies, case series, case reports, and reviews were excluded. Moreover, studies conducted on children or teenagers, peri-implant diseases, active gingivitis and/or periodontitis, and no hygiene interruption were also excluded.

\section{Screening process}

Two independent reviewers (C.M. and P.A.) conducted the search and screening process. Analysis 
Table 1. Systematic search strategy (PICO).

\begin{tabular}{|c|c|}
\hline Parameter & Search strategy \\
\hline Population & $\begin{array}{c}\text { "Humans" [MeSH Terms] OR "Humans" [Text Word] OR "Adults" [MeSH Terms] OR "Adults" [Text Word] "Oral } \\
\text { Health" [MeSh Terms] OR "Oral Health" [Text Word] OR "Gingivitis" [MeSh Terms] "Gingivitis" [Text Word] OR } \\
\text { "Experimental" [All Fields] }\end{array}$ \\
\hline Intervention & $\begin{array}{c}\text { "Probiotic" [MeSH Terms] OR "Probiotic" [Text Word] OR "Therapy" [subheading] OR "Therapeutics"[MeSH } \\
\text { Terms] OR "Therapy" [Text Word] }\end{array}$ \\
\hline Comparisons & $\begin{array}{c}\text { "Placebo" [MeSH Terms] OR "Placebo" [Text Word] OR "Lactobacillus" [MeSH Terms] OR "Lactobacillus" [Text } \\
\text { Word] OR "Cultured milk product" [Mesh Terms] OR "Cultured milk product" [Text Word] OR "Ice cream" [Mesh } \\
\text { Terms] OR "Ice cream" [Text Word] OR "Cheese" [Mesh Terms] OR "Cheese" [Text Word] OR "Yogurt" [Mesh } \\
\text { Terms] OR Yogurt [Text Word] OR "Lozenge" [All Fields] "Tablets" [MeSH Terms] OR "Tablets" [Text Word] OR } \\
\text { "Biofilm" [MeSH Terms] OR "Biofilm" [Text Word] OR "Dental Plaque" [MeSH Term] OR "Dental Plaque" [Text } \\
\text { Word] OR OR "Colonization" [All Fields] OR "Microbiota" [MeSH Terms] OR "Microbiota" [Text Word] OR } \\
\text { "Cytokines" [MeSH Terms] OR "Cytokines" [Text Word] }\end{array}$ \\
\hline Outcomes & $\begin{array}{l}\text { "Disease" [MeSH Terms] OR "Disease" [Text Word] OR "Activity [Text Word] OR "Inflammation" [MeSH Terms] } \\
\text { OR "Inflammation" [Text Word] OR "Bleeding" [Text Word] OR "Immunomodulation" [MeSH Terms] OR } \\
\text { "immunomodulation" [Text Word] OR "Biomarkers" [MeSH Terms] OR "Biomarkers" [Text Word] }\end{array}$ \\
\hline Study design & Randomized Clinical trial and Controlled Clinical trial: Follow up until 1 year. \\
\hline Search combination & \# 1 AND \#2 AND \#3 \\
\hline Database search & March to July 2018 \\
\hline Language & English \\
\hline Electronic database & PubMed/MEDLINE, Cochrane Central Register of Controlled Trials, Web of Science and Trip, Grey literature \\
\hline
\end{tabular}

of titles and abstracts was performed, followed by full-text article selection, analyzed according to eligibility criteria (inclusion/exclusion). Possible disagreements were resolved in concession meetings.

\section{Quality assessments}

The risk of bias was independently assessed by two authors (E.B. and K.V.) using the Cochrane Collaboration's tool. ${ }^{19}$ The analysis of each study was based on the following six criteria: sequence generation, allocation concealment, blinding, incomplete outcome data, selective outcome, and other sources of bias. Studies were rated at low, medium, or high risk of bias, when they met all, all except one, or all except two or more criteria, respectively.

\section{Data extraction}

Data were extracted (by D.L. and K.V), and a standardized form was used to record the following: authors, study design, clinical parameters (bleeding on probing - BOP, plaque index - PI, gingival index $\mathrm{GI}$, probing depth - PD, interproximal plaque index IPI, papilla bleeding index - PBI), gingival crevicular fluid volume - GCF, biomolecular parameters IL-1 $\beta$, IL-6, IL-8, IL-10,IL-18, TNF- $\alpha$, MIP-1 $\beta$, matrix metalloproteinase-8 (MMP-8), prostaglandin E2 (PGE2), nitrite/nitrate, matrix metalloproteinase-3 (MMP-3), polymorphonuclear elastase (PMN elastase), myeloperoxidase (MPO), microbiological findings, stage of the disease, probiotic administration, follow-up, and mean difference between baseline and final follow-up.

\section{Statistical analysis}

The positive effect of probiotics and the follow-up period of the included studies were calculated by estimating the intervention that was expressed in mean difference (MD) and $\mathrm{p}<0.05$. In this review, there was no possibility of performing a meta-analysis, due to the considerable heterogeneity between the studies.

\section{Results}

\section{Literature search}

The initial search resulted in 192 titles from PubMed/MEDLINE, 0 titles from Cochrane Central 
Register of Controlled Trials, 6 from Web of Science, and 47 from TRIP; a total of 245 titles. On the first title analysis, 123 duplicates were excluded. After abstract screening, 107 were excluded. Fifteen full-text articles were selected. After careful reading, ten studies were excluded as they failed to conform to the eligibility criteria of this review. Finally, five studies published between 2009 and 2017, were included in this systematic review (Figure). ${ }^{9,20,21,22,23}$

\section{Additional analysis}

The $\mathrm{k}$ concordance value for the two reviewers was $100 \%$ for the potential articles to be included (titles and abstracts) and for the articles selected; this indicated substantial concordance for the potential articles and 'perfect' agreement, $\mathrm{k}=1 .^{14}$

\section{Study characteristics}

The characteristics of the included studies are presented in Table 2. Four studies were RCTs ${ }^{9,20,21,22}$ and one study was a $\mathrm{CCT}^{23}$ comparing the use of probiotics (test group) with placebo (control group). The number of participants ranged from $18^{20}$ to $51,{ }^{9}$ with a mean of 36.2 participants. The mean follow-up period was 11.6 days (range 4-21 days). The included studies assessed whether daily oral administration of probiotic bacteria could influence the inflammatory response and the composition of supragingival plaque in an experimental gingivitis model. All studies evaluated PI and GCF;, ${ }^{9} 20-23$ three studies assessed PI, BOP, GI, and GCF;,20,21,22 one study evaluated $\mathrm{PBI}^{9}$ and IPI; and one study evaluated PD. ${ }^{22}$ The test groups from two studies received a milk drink containing Lactobacillus casei Shirota, ${ }^{9,23}$ two

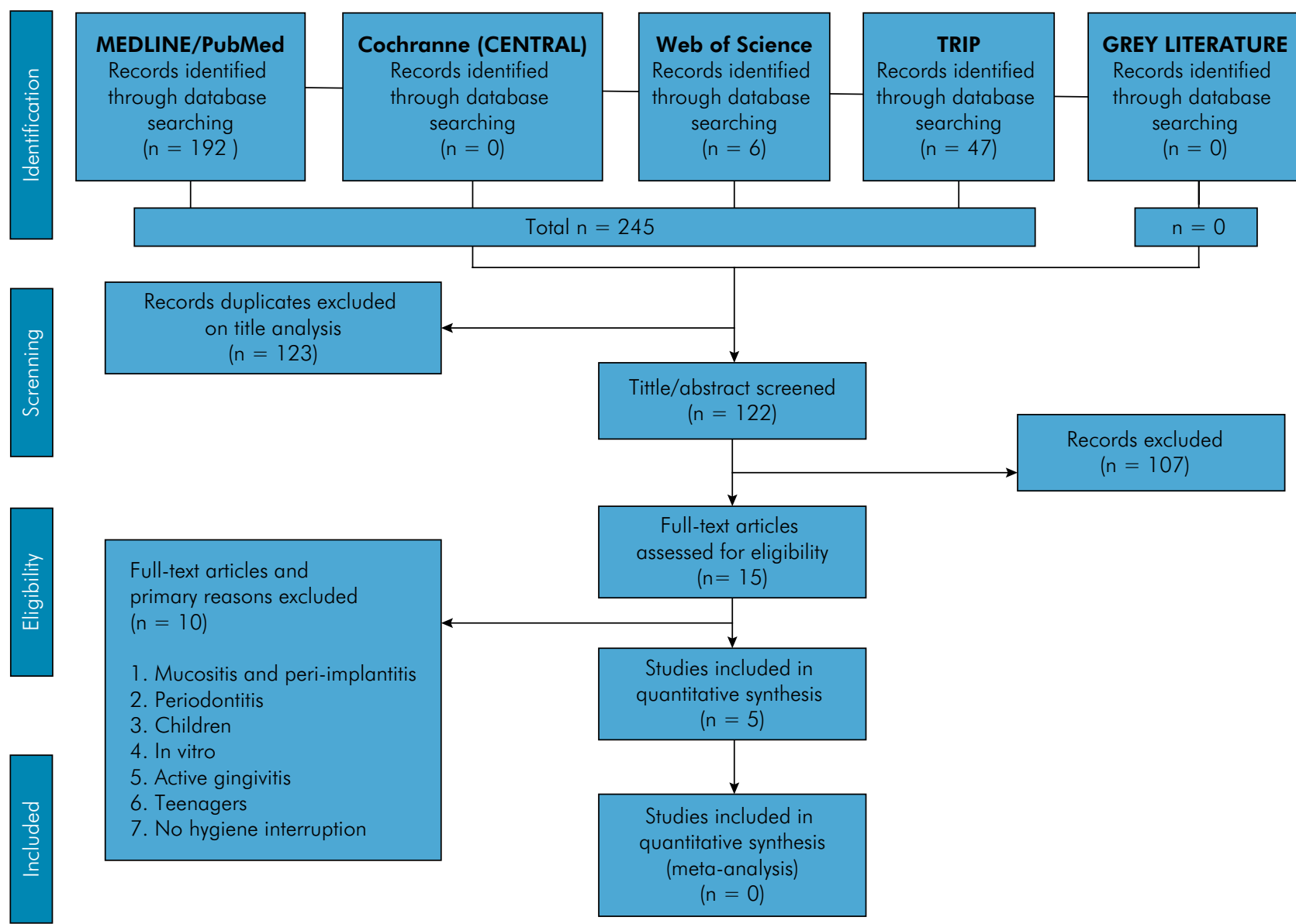

Figure. Flow diagram of the screening and selection process. 


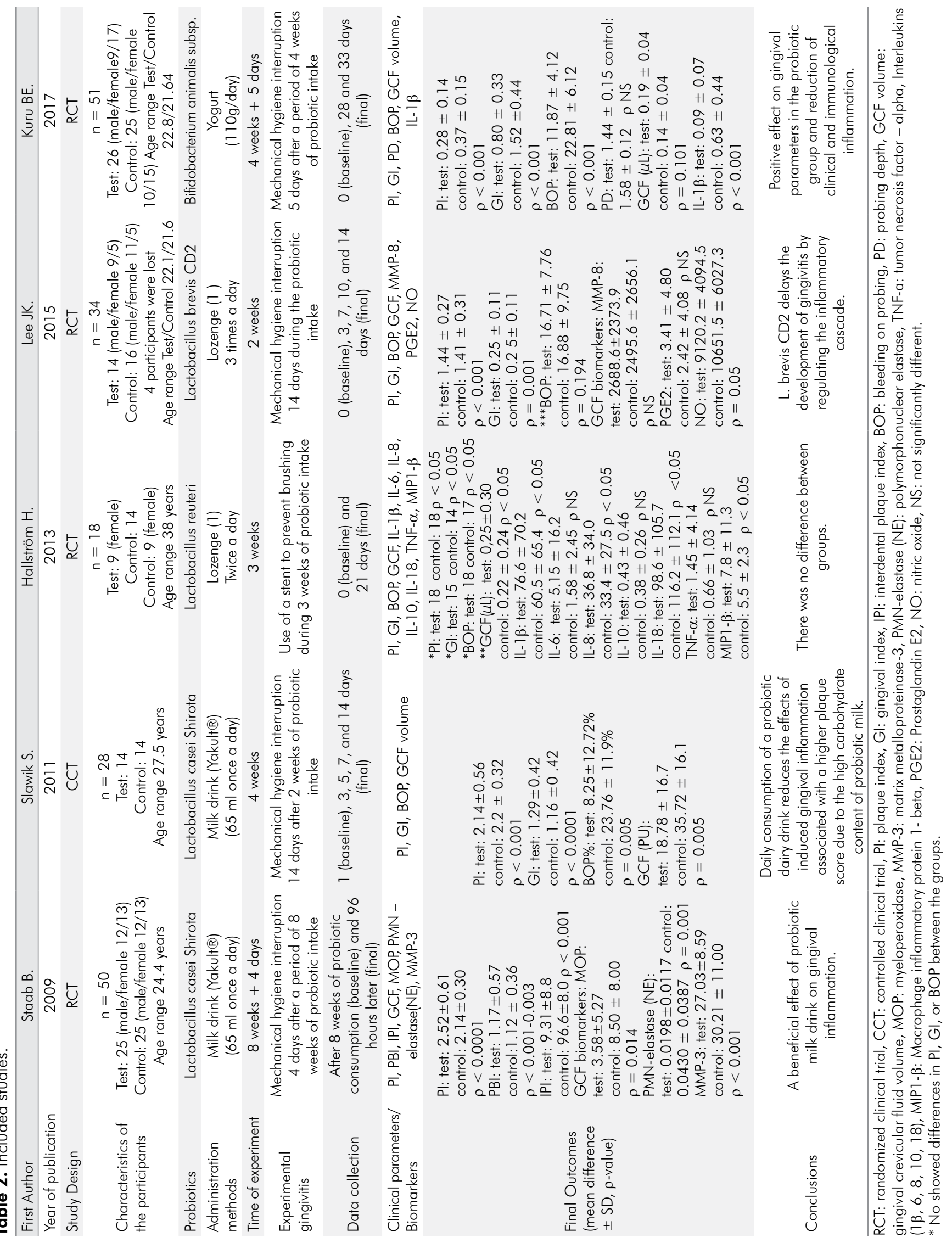


studies received lozenges containing Lactobacillus reuteri and Lactobacillus brevis $\mathrm{CD} 2,{ }^{20,21}$ and one study received Yogurt containing Bifidobacterium animalis subsp. lactis DN-173010.22

The studies conducted by Hallstrom et al. ${ }^{20}$ and Lee et al. ${ }^{21}$ evaluated the following clinical parameters: PI, GI, and BOP. In both studies, the results showed that there was an increase in PI, IG, and BOP from the baseline to the final follow-up, with no inter-group difference. However, Lee's study revealed that BOP was higher in the placebo group than in the probiotic group. ${ }^{21}$ In both studies, the subjects abstained from oral hygiene for two weeks. ${ }^{20,21}$ In the study by Lee et al., ${ }^{21}$ the participants refrained from tooth brushing, while Hallstrom et al. ${ }^{20}$ used an acrylic stent on the teeth involved in the study to prevent accidental cleaning.

Studies by Kuru et al. ${ }^{22}$ and Slawik et al..$^{23}$ have shown similar results for PI and GI. The indices showed a comparable increase in test and control groups. However, the BOP showed a significant increase in the control group. Kuru et al. ${ }^{22}$ was the only study that evaluated PD and found an increase in the control group. Staab et al. ${ }^{9}$ evaluated the interproximal plaque index (IPI) and, unlike the other studies, concluded that there was greater bleeding in the test group.

All studies included in this review showed a smaller change in GCF volume in the presence of probiotic. ${ }^{9,20,21,22,23}$ However, four studies ${ }^{9,20,21,22}$ analyzed specific biomarkers of inflammation, such as MMP-8, prostaglandin E2, nitrite/nitrate conversion, ${ }^{21}$ PMN-elastase, MPO and MMP- $3,{ }^{9}$ IL- $1 B,{ }^{22}$ IL-1ß, IL-6, IL-8, IL-10, IL-18, TNF- $\alpha$, and MIP-1B. ${ }^{20}$ In the evaluation of the biomarkers IL-1B, IL-18, MMP-3, and MPO, three studies showed increased fluid volume in the control group when compared to the test group. ${ }^{9,20,22}$ The concentrations of the biomarkers MIP-1B, IL-8 ${ }^{20}$ and prostaglandin E2 ${ }^{21}$ were lower in both groups between the baseline and the final follow-up. However, the biomarkers TNF $\alpha$, IL-6, IL-10 ${ }^{20}$, MMP- $8,{ }^{21}$ and PMN- elastase ${ }^{9}$ showed no intergroup difference.

One study examined the changes in the microbiological profile of supragingival plaque and concluded that the number of bacteria increased in both groups, mainly Fusobacterium nucleatum and Veillonella parvula. Additionally, the concentration of Streptococcus oralis was higher only in the test group. The bacteria Tannerella forsythia, Streptococcus mutans, and Lactobacillus fermentum were hardly identified in the samples. ${ }^{20}$

\section{Quality analysis}

The quality analysis of RCTs and CCT included in the study are shown in Table 3. Five studies showed a low risk of bias, ${ }^{9,20-23}$ two studies ${ }^{20,22}$ met all the criteria described in the Cochrane Collaboration's tool, ${ }^{14}$ and three studies scored negatively, ${ }^{9,21,23}$ one in each question. No study used the CONSORT statement guidelines. ${ }^{24}$

It is important to emphasize that the ELISA technique used by Staab et al. ${ }^{9}$ and Kuru et al. ${ }^{22}$ to analyze biomarkers may have been compromised by the low sensitivity of the method and the limited amount of fluid. Hallström et al. ${ }^{20}$ used the DNA-DNA Checkerboard hybridization method to analyze existing bacteria. Although this technique is quick

Table 3. Quality assessment of included studies.

\begin{tabular}{|c|c|c|c|c|c|}
\hline $\begin{array}{l}\text { First author and year of publication/ } \\
\text { Quality assessment }\end{array}$ & $\begin{array}{l}\text { Staab et al. }{ }^{9} \\
2009\end{array}$ & $\begin{array}{c}\text { Slawik et al. }{ }^{23} \\
2011\end{array}$ & $\begin{array}{l}\text { Hallström et al. }{ }^{20} \\
2013\end{array}$ & Lee et al. ${ }^{21} 2015$ & Kuru $^{22}$ et al. 2017 \\
\hline Adequate sequence generation & Yes & Yes & Yes & Yes & Yes \\
\hline Allocation concealment & Yes & No & Yes & Yes & Yes \\
\hline Blinding & No & Yes & Yes & Yes & Yes \\
\hline Incomplete outcome data addressed & Yes & Yes & Yes & No & Yes \\
\hline Selective outcome reporting & Yes & Yes & Yes & Yes & Yes \\
\hline Free of other sources of bias & Yes & Yes & Yes & Yes & Yes \\
\hline
\end{tabular}


and sensitive, it may demonstrate false-positive results leading to a risk of bias.

\section{Discussion}

This study systematically reviewed randomized clinical trials and a controlled clinical trial on the effect of probiotics on experimental gingivitis. The included studies showed different results. ${ }^{9,20,21,22,23}$ It was necessary to interrupt mechanical oral hygiene procedures to evaluate the parameters indicative of the host inflammatory response, such as the bleeding frequency of the gingiva and the gingival crevicular fluid volume. ${ }^{23}$ Lee et al. ${ }^{21}$ advised the volunteers to discontinue tooth brushing. Hallström et al. ${ }^{20}$ used an acrylic stent on the involved teeth to prevent accidental cleaning. This methodology may have influenced the clinical results obtained because of stent inadequacies or biofilm disruption by tooth brushing in adjacent areas.

All studies highlighted the immediate effects of probiotics during the non-brushing period. $9,20,21,22,23$ However, for probiotics to be effective in treatment or prevention, a minimum concentration of $1 \times 10^{8} \mathrm{CFU}$ should be administered.$^{25}$ In addition, the administration of probiotic can affect its immunomodulatory effect, since a constant issue in the development of functional foods is the functionality of bioactive cultures. ${ }^{26}$

Two studies ${ }^{20,21}$ used probiotic-containing lozenges, one contained Lactobacillus reuteri (ATCC55730 and ATCC PTA5289; $1 \times 10^{8} \mathrm{CFU}$ of each strain) twice daily, and another contained Lactobacillus brevis CD2 three times a day. ${ }^{21}$ Only one study used a milk drink (Yogurt) containing $\geq 10^{8}$ colony forming units (CFU)/g Bifidobacterium animalis subsp., once a day. ${ }^{22}$ Two other studies used a dairy drink containing Lactobacillus casei Shirota. ${ }^{9,23}$ The studies that used probiotics in the form of milk drinks showed better results with delayed development of gingivitis, demonstrated as a reduction of papillary bleeding and bleeding on probing, in addition to a moderate increase in GCF volume. ${ }^{9,22,23}$ However, they showed a comparable increase in biofilm in both groups, an observation that can be explained by the amount of carbohydrate present in the probiotics. ${ }^{922,23}$ The use of probiotic lozenges showed less expressive differences between groups. ${ }^{20,21}$ The survival of probiotic microorganisms can be affected by several factors such as the composition of the food matrix, $\mathrm{pH}$, carbon source, exposure to oxygen, and variation of the time-temperature binomial during processing and storage. ${ }^{26,27}$ In the present review, none of the studies evaluated the technological aspects of the lozenges and the viability of the probiotic microorganisms during the storage period. Our review suggested that the probiotic drink showed better results on experimental gingivitis than the probiotic lozenges. ${ }^{920,21,22,23}$ Functional foods, such as yogurt and milk, improved survival of microorganisms during the storage and the fermentation process enabling higher counts of probiotics in food. ${ }^{12,26}$

All studies presented differences in clinical response to probiotics due to the form of administration, dosage, time, and probiotic strains. ${ }^{9,20-23}$ Hallström et al. ${ }^{20}$ concluded that there was no difference in the clinical levels of IP, GI, and BOP between the groups. The study by Lee et al. ${ }^{21}$ used lozenges and found less bleeding in the placebo group, while the other clinical parameters did not differ. However, in the studies by Staab et al., ${ }^{9}$ Kuru et al., ${ }^{22}$ and Slawik et al., ${ }^{23}$ PI, GI, and BOP were lower in the test group, proving that the probiotic produced positive clinical results. It is important to emphasize that gingival bleeding is a sensitive and reliable clinical indicator of gingival inflammation. ${ }^{28}$

The inflammatory response was also assessed by GCF volume and its biomarkers. The study conducted by Slawik et al. ${ }^{23}$ was the only one to evaluate fluid volume. ${ }^{23}$ The results were comparable to other studies, 9,20,21,22 in which there was a significant increase in fluid volume in the control group. The IL-1B, IL-18, MMP-3, and MPO biomarkers showed higher levels in the placebo group than in the test group. Other biomarkers such as TNF $\alpha$, IL-6, IL-10, MMP8 , and PMN elastase did not present differences between the groups. ${ }^{9,20,21,22}$ The GCF biomarker concentrations indicated a positive probiotic effect on the immunomodulatory host response. The results of the studies suggested that probiotics delayed the development of experimental gingivitis. ${ }^{9,21,22,23}$

The only study that evaluated the microbiological profile of supragingival plaque showed that the 
number of bacteria increased in both groups. Bacteria Streptococcus oralis and Actinomyces naeslundii were the most prevalent. Fusobacterium nucleatum and Veillonella parvula grew in both groups. Streptococcus oralis grew only in the probiotic group, while Tannerella forsythia, Streptococcus mutans, and Lactobacillus fermentum were hardly identified in both groups. ${ }^{20}$ Although the study by Kuru et al. ${ }^{22}$ did not perform a microbiological analysis, the antimicrobial properties of Bifidobacteria could have influenced the composition of the biofilm by inhibiting the periodontopathogens during the period of non-brushing. In contrast, a recent systematic review observed that probiotic bacteria attach to the oral tissues more strongly than pathogens, being able to compete for adhesion surfaces, thus producing a new biofilm. The authors concluded that probiotic use benefits the maintenance of oral health by decreasing the number of colony-forming units (CFU) of the oral pathogens. ${ }^{29}$

All studies included in this SR concluded that biomarker response patterns and modulation of inflammatory cytokines indicated a beneficial effect of probiotics in host response regulation, which is evidenced even with a gradual increase of plaque., ${ }^{9,2,21,22,23}$

The results of this review should be interpreted with caution. The RCTs and CCT included in this study had short follow-up periods. Additionally, the studies did not analyze the microbiome. The quantitative and qualitative method of analysis offers greater accuracy in the evaluation of beneficial and harmful periodontal bacteria. Studies based on microbiome analysis techniques may explain dysbiosis and the anti-inflammatory action of the probiotics.

\section{Conclusions and clinical implications}

The results of the analyzed studies using probiotics in experimental gingivitis showed a slight improvement in clinical parameters. The GCF volume significantly decreased in the presence of probiotics in experimental gingivitis compared to the placebo group. The results indicated that the positive effect of probiotics were due to the modulation of the host response, not the anti-plaque effect. However, the available evidence presented heterogeneity between the type of study, type of probiotic, dosage, administration method, and non-brushing period. Therefore, randomized clinical trials are needed to elucidate the mechanisms of probiotic action and develop better delivery systems.

\section{Acknowledgments}

This study was financed in part by the Coordenação de Aperfeiçoamento de Pessoal de Nível Superior Brasil (CAPES) - Finance Code 001.

\section{References}

1. Nagappan N, John J. Antimicrobial efficacy of herbal and chlorhexidine mouth rinse: a systematic review. J Dent Med Sci. 2012 NovDec;2(4):5-10. https://doi.org/10.9790/0853-0240510

2. Parwani SR, Parwani RN, Chitnis PJ, Dadlani HP, Prasad SV. Comparative evaluation of anti-plaque efficacy of herbal and 0.2\% chlorhexidine gluconate mouthwash in a 4-day plaque re-growth study. J Indian Soc Periodontol. 2013 Jan;17(1):72-7. https://doi.org/10.4103/0972-124X.107478

3. Food And Agriculture Organization OF THE United Nations - FAO, World Health Organization - WHO. Guidelines for the evaluation of probiotics in food. London Ontario; 2002 [cited 2019 Feb 15]. Available from: http://who.int/foodsafety/fs_management/en/probiotic_ guidelines.pdf

4. Morales A, Gandolfo A, Bravo J, Carvajal P, Silva N, Godoy C, et al. Microbiological and clinical effects of probiotics and antibiotics on nonsurgical treatment of chronic periodontitis: a randomized placebo- controlled trial with 9-month follow-up. J Appl Oral Sci. 2018 Jan;26(0):e20170075. https://doi.org/10.1590/1678-7757-2017-0075

5. Burton JP, Drummond BK, Chilcott CN, Tagg JR, Thomson WM, Hale JD, et al. Influence of the probiotic Streptococcus salivarius strain M18 on indices of dental health in children: a randomized double-blind, placebo-controlled trial. J Med Microbiol. 2013 Jun;62(Pt 6):875-84. https://doi.org/10.1099/jmm.0.056663-0

6. Krasse P, Carlsson B, Dahl C, Paulsson A, Nilsson A, Sinkiewicz G. Decreased gum bleeding and reduced gingivitis by the probiotic Lactobacillus reuteri. Swed Dent J. 2006;30(2):55-60. 
7. Mayanagi G, Kimura M, Nakaya S, Hirata H, Sakamoto M, Benno Y, et al. Probiotic effects of orally administered Lactobacillus salivarius WB21-containing tablets on periodontopathic bacteria: a double-blinded, placebo-controlled, randomized clinical trial. J Clin Periodontol. 2009 Jun;36(6):506-13. https://doi.org/10.1111/j.1600-051X.2009.01392.x

8. Montero E, Iniesta M, Rodrigo M, Marín MJ, Figuero E, Herrera D, et al. Clinical and microbiological effects of the adjunctive use of probiotics in the treatment of gingivitis: a randomized controlled clinical trial. J Clin Periodontol. 2017 Jul;44(7):708-16. https://doi.org/10.1111/icpe.12752

9. Staab B, Eick S, Knöfler G, Jentsch H. The influence of a probiotic milk drink on the development of gingivitis: a pilot study. J Clin Periodontol. 2009 Oct;36(10):850-6. https://doi.org/10.1111/j.1600-051X.2009.01459.x

10. Riccia DN, Bizzini F, Perilli MG, Polimeni A, Trinchieri V, Amicosante G, et al. Anti-inflammatory effects of Lactobacillus brevis (CD2) on periodontal disease. Oral Dis. 2007 Jul;13(4):376-85. https://doi.org/10.1111/j.1601-0825.2006.01291.x

11. Twetman S, Stecksén-Blicks C. Probiotics and oral health effects in children. Int J Paediatr Dent. 2008 Jan;18(1):3-10. https://doi.org/10.1111/j.1365-263X.2007.00885.x

12. Champagne CP, Cruz AG, Daga C. Strategies to improve the functionality of probiotics in supplements and foods. Curr Opin Food Sci. 2018 Aug;22:160-6. https://doi.org/10.1016/i.cofs.2018.04.008

13. Moher D, Shamseer L, Clarke M, Ghersi D, Liberati A, Petticrew M, et al. Preferred reporting items for systematic review and meta-analysis protocols (PRISMA-P) 2015 statement. Syst Rev. 2015 Jan;4(1):1. https://doi.org/10.1186/2046-4053-4-1

14. Higgins JP, Green S. Cochrane Handbook for systematic reviews of interventions 5.1.0; 2011 [cited 2018 April 10]. Available from: www.cochrane-handbook.org

15. Moher D, Liberati A, Tetzlaff J, Altman DG. Preferred reporting items for systematic reviews and meta-analyses: the PRISMA statement.. Ann Intern Med. 2009 Aug;151(4):264-9. https://doi.org/10.7326/0003-4819-151-4-200908180-00135

16. Schardt C, Adams MB, Owens T, Keitz S, Fontelo P. Utilization of the PICO framework to improve searching PubMed for clinical questions. BMC Med Inform Decis Mak. 2007 Jun;7(1):16. https://doi.org/10.1186/1472-6947-7-16

17. Grey Literature Report [homepage]. New York: The New York Academy of Medicine; 2018 [cited 2018 October 10]. Available from: http://www.greylit.org

18. Open Grey [homepage]. Open Grey in Europe; 2018 [cited 2018 October 10]. Available from: http://www.opengrey.eu

19. Higgins JP, Altman DG, Gøtzsche PC, Jüni P, Moher D, Oxman AD, et al.; Cochrane Bias Methods Group; Cochrane Statistical Methods Group. The Cochrane Collaboration's tool for assessing risk of bias in randomised trials. BMJ. 2011 Oct;343 oct18 2:d5928. https://doi.org/10.1136/bmi.d5928

20. Hallström H, Lindgren S, Yucel-Lindberg T, Dahlén G, Renvert S, Twetman S. Effect of probiotic lozenges on inflammatory reactions and oral biofilm during experimental gingivitis. Acta Odontol Scand. 2013 May-Jul;71(3-4):828-33. https://doi.org/10.3109/00016357.2012.734406

21. Lee JK, Kim SJ, Ko SH, Ouwehand AC, Ma DS. Modulation of the host response by probiotic Lactobacillus brevis CD2 in experimental gingivitis. Oral Dis. 2015 Sep;21(6):705-12. https://doi.org/10.1111/odi.12332

22. Kuru BE, Laleman I, Yalnızoğlu T, Kuru L, Teughels W. The influence of a Bifidobacterium animalis probiotic on gingival health: a randomized controlled clinical trial. J Periodontol. 2017 Nov;88(11):1115-23. https://doi.org/10.1902/jop.2017.170213

23. Slawik S, Staufenbiel I, Schilke R, Nicksch S, Weinspach K, Stiesch M, et al. Probiotics affect the clinical inflammatory parameters of experimental gingivitis in humans. Eur J Clin Nutr. 2011 Jul;65(7):857-63. https://doi.org/10.1038/ejcn.2011.45

24. Schulz KF, Altman DG, Moher D. CONSORT 2010 Statement: updated guidelines for reporting parallel group randomised trials. BMC Med. 2010 Mar;8(1):18. https://doi.org/10.1186/1741-7015-8-18

25. Witzler JJ. Development of a potential probiotic lozenge [Dissertation]. Araraquara, SP: Universidade Estadual Paulista; 2018.

26. Champagne CP, Fustier P. Microencapsulation for the improved delivery of bioactive compounds into foods. Curr Opin Biotechnol. 2007 Apr;18(2):184-90. https://doi.org/10.1016/i.copbio.2007.03.001

27. Foligné B, Daniel C, Pot B. Probiotics from research to market: the possibilities, risks and challenges. Curr Opin Microbiol. 2013 Jun;16(3):284-92. https://doi.org/10.1016/j.mib.2013.06.008

28. Chapple IL, Mealey BL, Dyke TE, Bartold PM, Dommisch H, Eickholz P, et al. Periodontal health and gingival diseases and conditions on an intact and a reduced periodontium: consensus report of workgroup 1 of the 2017 World Workshop on the Classification of Periodontal and Peri-Implant Diseases and Conditions J Periodontol. 2018 Jun;45(Suppl 20):S68-77. htttps://doi.org/10.1002/JPER.17-0719

29. Seminario-Amez M, López-López J, Estrugo-Devesa A, Ayuso-Montero R, Jané-Salas E. Probiotics and oral health: a systematic review. Med Oral Patol Oral Cir Bucal. 2017 May;22(3):e282-8. https://doi.org/10.4317/medoral.21494 